\title{
Giant Magnetoresistive Effects in a Single Element Magnetic Thin Film
}

\author{
J.F. Gregg, ${ }^{1}$ W. Allen, ${ }^{1}$ K. Ounadjela, ${ }^{2}$ M. Viret,${ }^{3}$ M. Hehn, ${ }^{2}$ S. M. Thompson, ${ }^{4}$ and J. M. D. Coey ${ }^{3}$ \\ ${ }^{1}$ Clarendon Laboratory, Parks Road, Oxford, OX1 3PU, United Kingdom \\ ${ }^{2}$ IPCMS, rue du Loess, 67037 Strasbourg Cedex, France \\ ${ }^{3}$ Department of Pure and Applied Physics, Trinity College, Dublin 2, Ireland \\ ${ }^{4}$ Department of Physics, University of York, Heslington, York YO1 5DD, United Kingdom
}

(Received 24 April 1996)

\begin{abstract}
By exploiting the simplicity of a novel transport measurement on a ferromagnetic striped domain structure in a thin film of cobalt, we report the first direct observation of ferromagnetic domain wall scattering and what we believe to be the first clear indication of giant magnetoresistive effects in a homogeneous magnetic system. (The colossal MR effect, while seen in homogeneous materials, is believed to originate from a charge ordering phenomenon [see, for example, Y. Tokura et al., J. Appl. Phys. 79, 5288 (1996), and references therein], and is therefore distinct from GMR, which is an effect arising from spin dependent scattering.) A new model is proposed to describe these observations which highlights the crucial role played by electron spin precession in determining the electrical transport properties of magnetic interfaces. [S0031-9007(96)00854-X]
\end{abstract}

PACS numbers: 73.50.Jt, 72.15.Gd, 75.60.Ch, 75.70.Kw

Conventional electronics has ignored the spin of the electron. Recently this omission has been partially redressed by intensive study of giant magnetoresistance (GMR) in magnetic multilayers and its explanation in terms of spin dependent electronic scattering [1,2]. It usually arises in heterogeneous magnetic systems such as magnetic trilayers [3], multilayer stacks consisting of alternating magnetic and nonmagnetic thin films $[4,5]$, or granular mixtures of magnetic and nonmagnetic metals [6-8]. The question has been informally discussed as to why GMR should not also occur in domain walls separating oppositely magnetized ferromagnetic domains, since the magnetic configuration closely resembles that of a magnetic trilayer, i.e., two regions of oppositely pointing magnetization separated by a thin interlayer (Fig. 1). Historically it has been difficult to generate incontrovertible data on domain wall scattering: papers such as Ref. [9] (where the interpretation is complex and relies on hypothesized domain structures which cause current-dependent sample resistance) serve to underline the need for a clear, unambiguous experiment to measure domain wall resistivity in ferromagnets.

The experiment described in this Letter attempts to fulfill this requirement. We will show that magnetoresistance of comparable magnitude to that associated with interfaces in GMR trilayers can arise from domain walls within a chemically homogeneous thin film of a ferromagnet with appropriate spin precession parameters. The experimental results are numerically inconsistent with the various models for domain wall scattering in the literature. We therefore advance a new model which, while it is not a straightforward transfer of the theory of current perpendicular to plane (CPP) GMR in magnetic thin film trilayers (as might naively be hoped from consideration of Fig. 1), nevertheless invokes many of the features of this phenomenon.
The sample consists of a $1000 \AA$ thick film of cobalt grown at $400{ }^{\circ} \mathrm{C}$ by molecular beam epitaxy onto a sapphire $\left(\mathrm{Al}_{2} \mathrm{O}_{3}\right)$ substrate with a ruthenium buffer layer $200 \AA$ thick and a $50 \AA$ ruthenium capping layer. From electron diffraction during growth and subsequent $\mathrm{x}$-ray diffraction, the structure is seen to be high crystalline quality hcp (0001) cobalt with the magnetic easy axis perpendicular to the film plane. In zero applied field the film adopts a maze configuration of perpendicularly magnetized domains [Fig. 2(a)]. The domains are about $900 \AA$ across-considerably smaller than in bulk metal where

a)

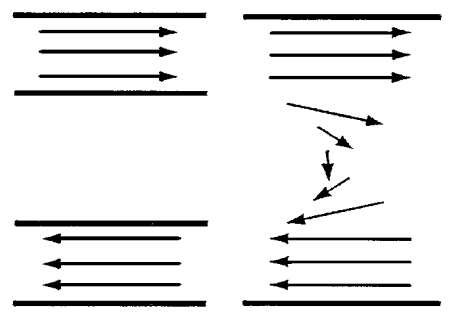

b)

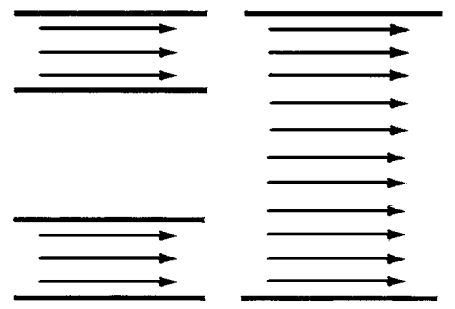

FIG. 1. Schematic illustration of the similarity between the magnetic geometry of a GMR trilayer and a ferromagnetic domain wall. (a) The relationship between an antiferromagnetically aligned trilayer and the presence of a domain wall. (b) The trilayer in its ferromagnetically aligned state, which corresponds to the absence of the domain wall in the ferromagnet. 


$$
5 \mu \mathrm{m}
$$
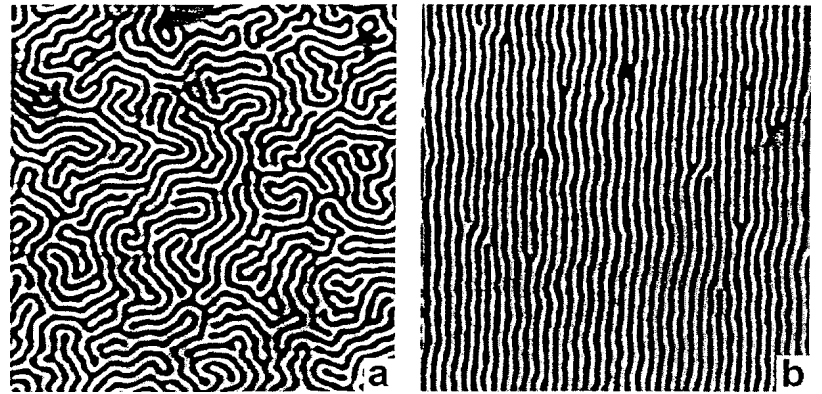

FIG. 2. Domain patterns for a $1000 \AA$ thick cobalt film as imaged by magnetic force microscopy. (a) The initial domain configuration in zero applied field. (b) The beautiful parallel stripe domain pattern which may be prepared by single domaining the film in a large magnetic field applied in the plane of the film, then demagnetizing the film by cycling the in-plane field.

they measure about $1 \mu \mathrm{m}$. On demagnetizing from inplane saturation with an in-plane magnetic field, the film retains an elegant memory of its former state [Fig. 2(b)]. The zero-field state is now a set of uniform stripe domains oriented parallel to the direction of the demagnetizing field; their magnetization is again perpendicular to the plane.

For the transport measurement, the electric current was applied perpendicular to the striped domain structure and a magnetic field was applied perpendicular to the film plane. In this geometry, the observed magnetoresistance is a consequence of spin dependent scattering at the domain walls. In particular, anisotropic magnetoresistance (AMR) is effectively suppressed since the current and the magnetization remain mutually perpendicular throughout the magnetic field sweep. There is a small in-plane remanance magnetization (about 20\% of the saturation magnetization) but its size is too small to correspond to the resistance changes observed. Moreover, it vanishes quickly on application of a perpendicular magnetic field, so it is probably responsible for the small accidents around zero field on our low field resistance plateau. By careful attention to the sample contact geometry the anomalous Hall effect is similarly suppressed.

The low field resistance plateau in the initial magnetoresistance curve of the stripe domain state [Fig. 3(a)] corresponds to the linear region of the magnetization curve [Fig. 3(d)] where domains parallel to the applied field grow at the expense of those oriented antiparallel. The resistance stays constant because domain wall density is conserved in this process. As the field is further increased, walls are destroyed and the resistance decreases steeply. That the resistance apparently saturates at much higher fields than the magnetization is an illusion caused by the survival to several tesla of bubble domains stabi- lized by surface defects. There may also be an additional contribution from magnon damping.

These measurements give $\Delta \rho_{\mathrm{Co}}=5.2 \times 10^{-9} \Omega \mathrm{m}$ for the additional resistivity of the domain wall material over that of the bulk cobalt. From measurements on nickel by a related experiment [10], we find $\Delta \rho_{\mathrm{Ni}}=1.2 \times 10^{-9} \Omega \mathrm{m}$. This disagrees with the factor of $\Delta \rho_{\mathrm{Co}} / \Delta \rho_{\mathrm{Ni}} \cong 75$ predicted by the established spin dependent potential model [11] for the ratio of the additional resistivities. The predictions of the diamagnetic effect model [11] are similarly adrift.

Moreover, the cobalt sample, the resistance measured per unit area of domain wall is $7.8 \times 10^{-17} \Omega \mathrm{m}^{2}$. This is similar to the magnetoresistance per interface pair $\left(3.8 \times 10^{-17} \Omega \mathrm{m}^{2}\right)$ in CPP CoCu multilayers exhibiting $17 \%$ room temperature GMR [12], and this similarity encourages exploration of a link between the phenomena. Paper I of Ref. [11] already proposes a theory which looks uncannily like the GMR two-spin-channel model in all but name. However, it takes no account of the precessional behavior of the carrier spins in the exchange field of the domain wall.

These observations suggest a new model of domain wall resistivity which, unlike previous approaches $[11,13]$, invokes the fundamentals of GMR spin dependent scattering theory together with a treatment, analogous to that of adiabatic fast passage in magnetic resonance, of the precessional behavior of the carriers. In it, the key to electrical scattering by domain walls is how well the precessional behavior of the carrier spin allows it to track the changing local exchange field direction as it traverses the wall. The spin tracks successfully if the angular rotation frequency of the exchange field in the frame of the spin is small compared to the precession frequency of the spin in the same exchange field (see Fig. 4). This is equivalent to the spin precession length being small compared to the domain wall thickness, i.e., to a large value of the parameter

$$
\xi=2 E_{\mathrm{ex}} d / h v_{F},
$$

where $v_{F}$ is the Fermi velocity, $d$ the domain wall thickness, and $E_{\text {ex }}$ the exchange energy.

Our model assumes two scattering terms for majority carriers in domain wall material, one of which exactly mirrors the spin dependent interface scattering mechanism in multilayer GMR while the other is equivalent to the spin dependent bulk scattering term. The first is the effective potential seen by the spin due to the wall on account of the changing spin deviation $\theta$ from the local exchange field direction whose amplitude is

$$
\theta_{0}=K h v_{F} / E_{\text {ex }} d \text {, }
$$

where $K$ depends on the shape of the Fermi surface. The second invokes the GMR mechanism by which carrier scattering depends on admixture of minority spin wave function and hence deviation from spin quantization 

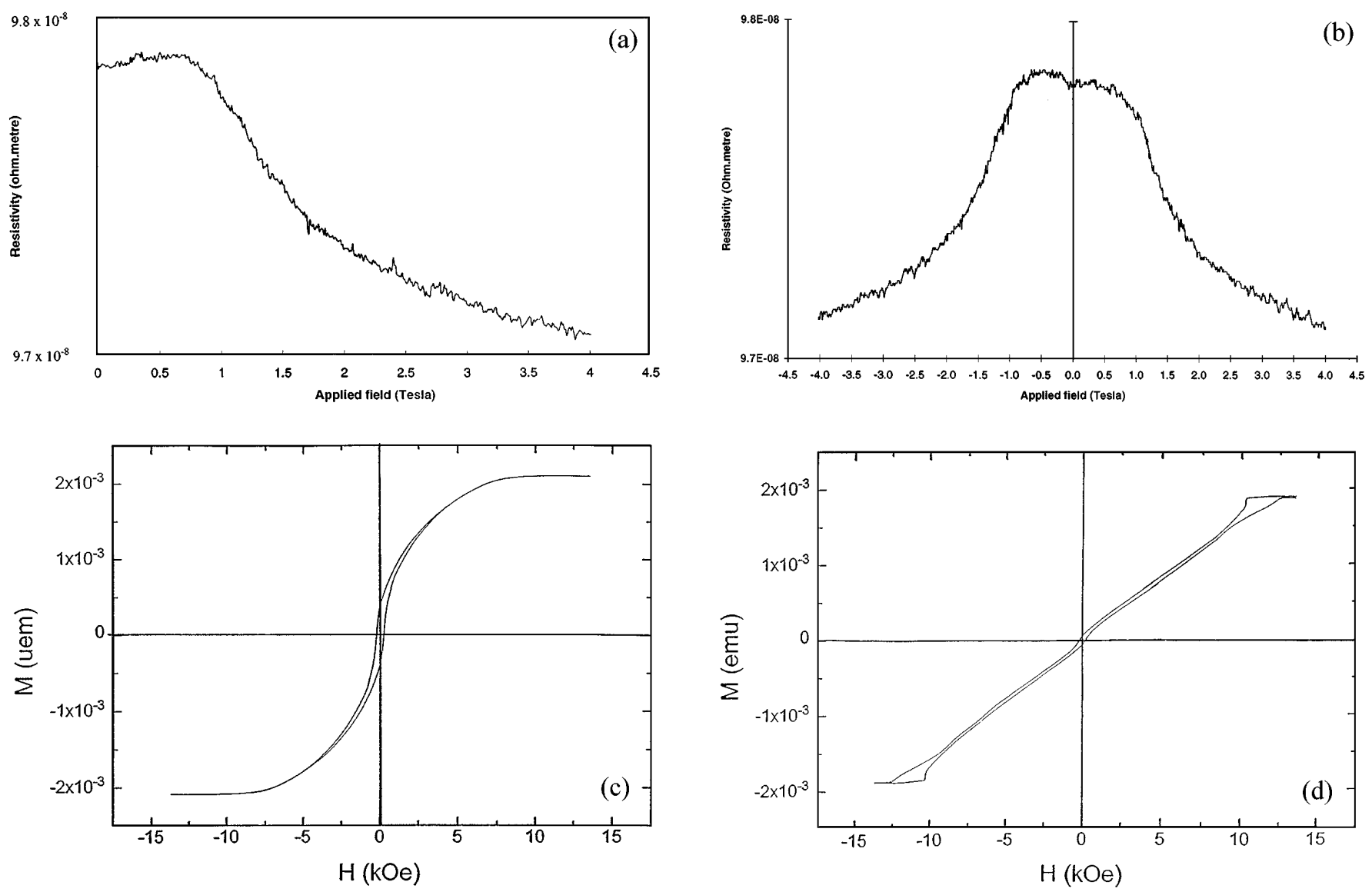

FIG. 3. (a) The resistivity of our $1000 \AA$ cobalt film as a function of magnetic field. The field is applied out of plane and the sample has been previously prepared by saturating and then demagnetizing with the field in plane so as to produce an array of parallel stripe domains [Fig. 2(b)]. The field sweep starts from this zero-field configuration. A resistance plateau is observed as the field is increased, followed by a decrease which sets in as the saturation field is approached and pairs of complementary domain walls begin to annihilate one another. (b) The resistivity as a function of magnetic field for both positive and negative magnetic field sweeps, indicating the field-even nature of the effect. The resistance plateau is again observed in low field. The small accidents in the vicinity of zero field are almost certainly small anisotropic magnetoresistance (AMR) contributions which arise from the small in-plane magnetic remanence which disappears rapidly as field is applied [see (c)]. It should be noted that, unlike (a), these data do not correspond to the simple striped domain pattern of Fig. 2(b). The scans start in large negative field where memory of the striped domain state has been almost completely effaced and proceed through zero (where a bubble domain configuration obtains) to large positive field. As discussed in the text, the resistivity observed is very similar to that seen in the CPP-like geometry corresponding to (a), owing to the conduction in the domain wall being in the diffusive limit where the mean free path is smaller than the wall width. (c),(d) The magnetization curves in plane and out of plane, respectively, for the same sample. The curve shapes bear the characteristic signature of a perpendicular magnetized multidomain structure. A small in-plane remanance is apparent (about $20 \%$ of the saturation magnetization) which disappears when small perpendicular fields are applied. This is probably the cause of the accidents near zero field on trace (b).

direction. Its scattering probability for any region of wall material is therefore determined by the instantaneous direction of the spin in question relative to the local exchange field in that part of the wall. Integrated over the Fermi surface and the body of the domain wall (the integral must include a depth of several spin diffusion lengths into the adjacent domains) both terms give rise to an additional resistivity for the domain wall material which varies as $\sin ^{2}(\theta / 2) \propto 1 / \xi^{2}$.

It should be noted that the model remains valid in the diffusive conduction limit when the mean free path is smaller than the domain wall thickness, since the macroscopic GMR depends on an integral over carrier phase space which is independent of whether the phase space trajectories are continuous or discontinuous. In CPP GMR the essential criterion is that the separation of the adjacent magnetic layers should be smaller than the spin diffusion length so that the magnetic information transfer between layers may be mediated by the carrier spin memory. The equivalent criterion in our case is more subtle and essentially requires that spin information should survive for a carrier displacement across the domain wall equivalent to the distance over which the spin diverges from the local exchange field direction. In short, the spin precession length should not be substantially greater than either the mean free path or the spin diffusion 
(a)

(b)

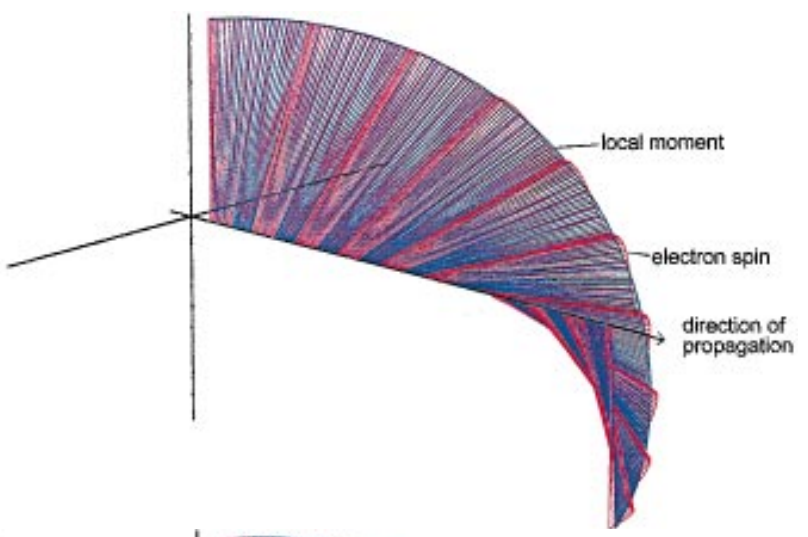

)

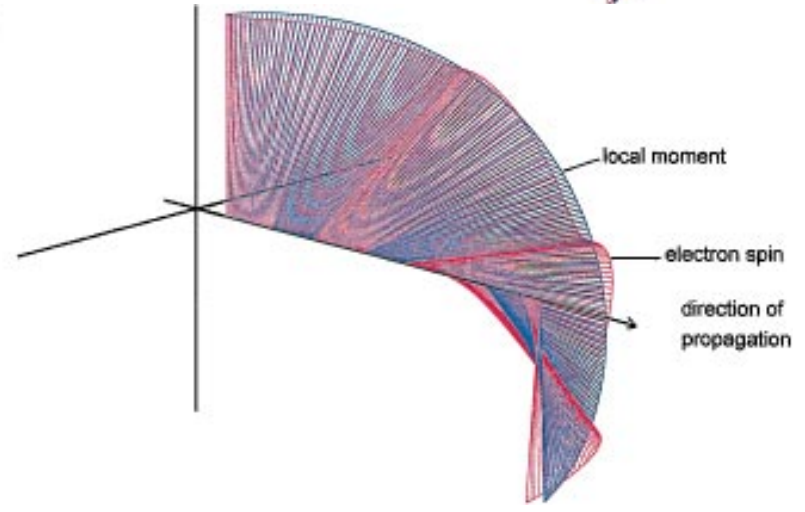

FIG. 4(color). The spin trajectory is shown for electrical carriers in transit through domain walls in a ferromagnet. The spin orientation is shown in red; the local exchange field variation is shown in blue. (a) The spin precessional behavior for a nickel domain wall while (b) shows the equivalent behavior for cobalt. The exchange energies used in the simulation were $1.0 \mathrm{eV}$ for $\mathrm{Co}$ and $0.3 \mathrm{eV}$ for $\mathrm{Ni}$, the respective wall thicknesses were 150 and $1000 \AA$, and the majority spin Fermi velocities employed were $10^{6} \mathrm{~m} / \mathrm{s}$, giving the respective tracking parameters as $\xi_{\mathrm{Co}}=7.3$ and $\xi_{\mathrm{Ni}}=14.5$. It should be noted that the higher value of $\xi_{\mathrm{Ni}}$ gives rise to better spin tracking, hence smaller deviation angle and lower domain wall resistivity in nickel than in cobalt.

length in the ferromagnetic metal: This condition obtains for the two materials discussed in this paper.

Incidentally, in the diffusive limit it follows that the "CPP-like" geometry is not essential to observe this effect, and this is indeed what we find experimentally as is apparent from the scans of Fig. 3(b).

This simple model explains satisfactorily our measured value $\Delta \rho_{\mathrm{Co}} / \Delta \rho_{\mathrm{Ni}}=4.3$ which agrees with the ratio $\left(\xi_{\mathrm{Ni}} / \xi_{\mathrm{Co}}\right)^{2}=4.0$ within the uncertainty of the parameters used to estimate $\xi$ and reflects the respective mistracking of the cobalt and nickel spins as illustrated in Fig. 4. As discussed above, the model is also capable of predicting the right order of magnitude for the absolute values of the magnetoresistance as well as their ratio for the two materials; however, attempting close quantitative agreement between domain walls and trilayers involving the same ferromagnetic material is difficult since modeling this numerically involves rather more variables which depend critically on film preparation, etc.

In conclusion, we believe this to be the first direct observation of domain wall scattering and, by inference, of the GMR effect in a film of pure ferromagnetic element. It underlines the importance of carrier spin precession in determining the electrical transport properties of magnetic metal interfaces. The effect has potential for spin commutation in the nascent field of spin electronic devices $[14,15]$ in which electric currents are injected and manipulated by labeling them with different electronic spin states.

This work was supported by the European Human Capital and Mobility programme, the British Council Alliance programme, EPSRC, and Leybold U.K. The authors are grateful to Professor A. Fert for asking the question which caused them to think of this experiment.

[1] T. Valet and A. Fert, Phys. Rev. B 48, 7099-7113 (1993).

[2] S. S. P. Parkin, Phys. Rev. Lett. 71, 1641-1644 (1993).

[3] B. Dieny, V.S. Speriosu, S. S. P. Parkin, B. A. Gurney, D. R. Wilhoit, and D. Mauri, Phys. Rev. B 43, 1297-1300 (1991).

[4] M. N. Baibich, J. M. Broto, A. Fert, F. Nguyen Van Dau, and F. Petroff, Phys. Rev. Lett. 61, 2472-2475 (1988).

[5] G. Binasch, P. Grünberg, F. Saurenbach, and W. Zinn, Phys. Rev. B 39, 4828-4830 (1989).

[6] A. E. Berkowitz, J. R. Mitchell, M. J. Carey, A. P. Young, S. Zhang, F.E. Spada, F. T. Parker, A. Hutten, and G. Thomas, Phys. Rev. Lett. 68, 3745-3748 (1992).

[7] J. Q. Ziao, J.S. Ziang, and C. L. Chien, Phys. Rev. Lett. 68, 3749-3752 (1992).

[8] J. A. Barnard, A. Waknis, M. Tan, E. Haftek, M. R. Parker, and M.L. Watson, J. Magn. Magn. Mater. 114, L230-232 (1992).

[9] G. R. Taylor, Acar Isin, and R. V. Coleman, Phys. Rev. 165, 621-631 (1968).

[10] M. Viret, D. Vignoles, D. Cole, J. M. D. Coey, W. Allen, D. S. Daniel, and J. F. Gregg, Phys. Rev. B 53, 8464-8468 (1996).

[11] G. G. Cabrera and L. M. Falicov, Phys. Status Solidi (b) 61, 539-549 (1974); 62, 217-222 (1974).

[12] M. A. M. Gijs, M.T. Johnson, A. Reinders, P.E. Huisman, R. J.M. van de Veerdonk, S. K. J. Lenczowski, and R. M. J. van Gansewinkel, Appl. Phys. Lett. 66, 18391841 (1995).

[13] L. Berger, J. Appl. Phys. 49, 2156-2161 (1978).

[14] M. Johnson, Science 260, 320 (1993).

[15] D. J. Monsma, J. C. Lodder, J. A. Popma, and B. Dieny, Phys. Rev. Lett. 74, 5260 (1995). 